\title{
LA EVALUACIÓN DE LA FORMACIÓN UNIVERSITARIA SEMIPRESENCIAL Y EN LÍNEA EN EL CONTEXTO DEL EEES MEDIANTE EL USO DE LOS INFORMES DE ACTIVIDAD DE LA PLATAFORMA MOODLE
}

\author{
(THE EVALUATION OF BLENDED AND ON-LINE UNIVERSITY TEACHING AND LEARNING \\ MODELS IN THE CONTEXT OF THE EUROPEAN HIGHER EDUCATION AREA (EHEA) BY MEANS \\ OF THE USE OF MOODLE PLATFORM ACTIVITY REPORTS)
}

\author{
Bonifacio Martín Galán \\ David Rodríguez Mateos \\ Universidad Carlos III de Madrid (España)
}

\section{RESUMEN}

La incorporación de las universidades españolas al Espacio Europeo de Educación Superior (EEES) ha impulsado nuevos modelos de evaluación de la actividad docente universitaria, recogidos en diversos programas nacionales de control, entre los que destaca el programa DOCENTIA de la ANECA. Sin embargo, en los nuevos contextos de formación semipresencial y a distancia ofertados en diversas titulaciones de nuestro país, la incorporación de plataformas informáticas para la gestión del aprendizaje posibilita la extracción y el tratamiento estadístico de los datos procedentes de los ficheros de actividad del sistema. Se presenta la experiencia de formación semipresencial en diversas titulaciones llevadas a cabo por la Universidad Carlos III de Madrid haciendo uso de la plataforma Moodle y la necesidad de recoger toda la información generada por este sistema como complemento y apoyo a la hora de establecer indicadores de utilidad para el proceso evaluativo de la docencia universitaria.

Palabras clave: Espacio Europeo de Educación Superior (EEES), evaluación de la docencia, enseñanza a distancia, sistemas de gestión del aprendizaje (LMS), registros de actividad en Moodle, tratamiento estadístico.

\section{ABSTRACT}

The incorporation of Spanish universities into the European Higher Education Area (EHEA) has given rise to new models of assessment for university teaching. These models are placed under the general heading "teaching performance programs", among which are the DOCENTIA program run by ANECA (the Spanish National Agency for Quality Assessment and Accreditation). Within the new context of 
blended and distance learning models, namely those that are currently being used for various university qualifications, the incorporation of information technology platforms in the management of learning makes it possible to extract data and to statistically analyze information using the system's "activity grids". We examine the experience of blended learning courses for various academic programs that are being held at Carlos III University, e.g. via the Moodle platform. This includes the need to gather all the information that is generated by this system, i.e. complementary and support materials for establishing quality indicators in the evaluation process of university teaching.

Keywords: European Higher Education Area (EHEA), evaluation of teaching quality, blended learning, Learning Management Systems (LMS), Moodle activity report, statistical treatment (of data).

Es un hecho irrefutable en nuestros días que el uso de las tecnologías de la información y la comunicación (TIC) y más recientemente, las tecnologías digitales y las plataformas de publicación web, han venido favoreciendo en estas últimas décadas la modernización de los modelos educativos tradicionales imperantes basados, por un lado, en la presencialidad del alumno y, por el otro, en la clase magistral del docente.

A este avance se le ha sumado, en el contexto de aplicación de los centros universitarios españoles, el decidido impulso que ha tomado en estos últimos años esta comunidad para su plena integración en el denominado Espacio Europeo de Educación Superior (EEES). Esta integración supone, entre otras muchas cosas, una profunda reflexión sobre las metodologías docentes a aplicar a fin de alcanzar el paradigma promulgado por este nuevo modelo de educación universitaria, en el que se pretende establecer un sistema basado en la adquisición de competencias y con una clara orientación hacia el aprendizaje del estudiante, sin excluir, con ello, el enfoque clásico basado en contenidos y horas lectivas.

Este aprendizaje debe posibilitar la adquisición por parte del alumno de las habilidades necesarias para un correcto desarrollo de la profesión elegida. Se asume que la adquisición de estas competencias y habilidades se debe realizar a través de un proceso de autoaprendizaje guiado y tutorizado, siendo, por tanto, imprescindible por parte del estudiante una participación mucho más activa que la mera asistencia a clase o el desarrollo, más o menos regular, de alguna actividad de carácter práctico (Oliveros, 2006).

Este concepto de aprendizaje constructivista debe ser sostenido por una estrategia pedagógica capaz de impulsar el cambio del paradigma docente universitario propuesto desde las instituciones europeas. A este respecto, la actual legislación en 
materia de enseñanzas universitarias oficiales en España ${ }^{1}$ apuntala estas ideas, al señalar que la reorganización educativa que se está produciendo debe responder no sólo a un cambio estructural sino, más bien, a un cambio en las metodologías docentes que centre el objetivo en el proceso de aprendizaje del estudiante, en un contexto que debe extenderse a lo largo de su vida. Así, estas nuevas metodologías deben poner en valor la motivación y el esfuerzo del estudiante para aprender (Area, 2006).

Otras ideas fundamentales en este nuevo contexto de ordenación de las enseñanzas universitarias son las de la diversidad y la flexibilidad, como respuesta a las demandas de las sociedades actuales en un contexto abierto y en constante transformación. Estos conceptos, enmarcados en el objetivo principal del presente trabajo, inciden directamente en la aplicación de otros modelos o tipos de enseñanza más allá de la tradicional clase magistral de carácter presencial y donde las tecnologías digitales toman todo su sentido. Comienzan, por tanto, a surgir nuevas modalidades formativas que recogen lo mejor del sistema tradicional y lo complementen con las nuevas técnicas y procedimientos propios de los modelos educativos no presenciales en entornos de red (principalmente en la Web), tales como el b-learning (blended learning o aprendizaje mixto), el e-learning (electronic learning o aprendizaje electrónico o a distancia) o, más recientemente, el m-learning (mobile learning o aprendizaje electrónico móvil).

En todos ellos, el elemento central para la construcción y la administración de las webs docentes y de las actividades de formación no presencial lo constituye una plataforma virtual de teleformación, a menudo referidas de innumerables maneras (Díaz-Antón y Pérez, 2005), pero habitualmente conocidas en su acepción anglosajona como CMS (Course Management System) o, de forma más habitual, LMS (Learning Management System).

Estos nuevos modelos de enseñanza-aprendizaje a distancia mediante el uso de las tecnologías Web aportan numerosos beneficios frente al modelo tradicional (independencia del tiempo y del espacio; comunicaciones interactivas; capacidades multimedia; incrementa las habilidades en la expresión escrita; reducción de costes; etc.), pero no están exentos de problemas y de detractores (Ahn y Han, 2005).

Sin embargo, es evidente que resulta un movimiento imparable pues, además de todo lo señalado, se cumple con otro de los preceptos fundamentales de toda esta normativa europea y nacional en el ámbito de la educación superior: que los sistemas de acceso y, por extensión, de formación docente, potencien la apertura 
hacia los estudiantes de otras áreas geográficas, tanto nacionales como de otros países del EEES.

Enmarcado en este contexto, el Departamento de Biblioteconomía y Documentación de la Universidad Carlos III de Madrid optó en el curso 20072008 por diversificar su oferta educativa para sus titulaciones de diplomatura y de licenciatura, ofreciendo, además de la clásica oferta presencial en su campus de Getafe, nuevos grupos bajo un modelo semipresencial en su campus de Colmenarejo, cuya herramienta básica es la plataforma Moodle para la enseñanza a distancia. En el curso 2008-2009, con la adaptación de las titulaciones oficiales de esta universidad al EEES, se incorporó a esta oferta académica en su doble modalidad presencial y semipresencial el nuevo Grado en Información y Documentación, así como varios grupos de un curso especial de adaptación a este grado para antiguos alumnos de la diplomatura (Iribarren et al., 2008). Finalmente, esta oferta se ha visto completada en el curso 2009-2010 con el lanzamiento de un nuevo postgrado oficial (máster universitario), en modalidad igualmente semipresencial, sobre bibliotecas y servicios de información digital. En el curso 2010-2011 se ha incorporado a esta oferta de titulaciones en modalidad semipresencial de esta universidad un grupo del Grado en Humanidades, haciendo igualmente uso de la plataforma Moodle como eje tecnológico central en su docencia.

\section{LA ACTIVIDAD DOCENTE UNIVERSITARIA EN EL EEES Y SU EVALUACIÓN}

La libertad y la autonomía que todas estas normas conceden a las universidades españolas para el diseño de sus titulaciones vienen acompañadas, sin embargo, de un mayor control y un alto nivel de supervisión por parte de la Administración pública. Así, es posible afirmar, como señala Murillo Torrecilla (2008) que el total de las universidades españolas han venido desarrollando, de un modo u otro, planes de evaluación del desempeño docente aunque con notables diferencias entre ellas.

Para tratar de armonizar y dar garantía a estos procesos, las universidades españolas han establecido sistemas de evaluación y acreditación, conocidos por el nombre formal de Sistema de Garantía de la Calidad, que supervisa la ejecución efectiva de esta nueva organización de las enseñanzas universitarias. Su estructura es piramidal, existiendo, en el caso español, un organismo central, la Agencia Nacional de Evaluación de la Calidad y Acreditación (ANECA) (http://www.aneca.es/), con el objetivo expreso de "contribuir a la mejora de la calidad del sistema de educación superior mediante la evaluación, certificación y acreditación de enseñanzas, 
profesorado e instituciones". En un nivel inferior, se sitúan las agencias propias de cada comunidad autónoma del país, dado que en la mayoría de ellas se tienen asumidas las competencias en materia de educación superior. En el nivel más bajo, se sitúan las propias instituciones universitarias, a las que corresponde, por tanto, el desarrollo de su sistema de garantía interna de la calidad.

La ANECA lleva a cabo la misión encomendada a través de diversos programas de actuación. Además del grupo de programas encaminados específicamente a la evaluación del profesorado como paso previo para su contratación por parte de las universidades, existe un gran grupo destinado específicamente a los programas orientados a la evaluación de las enseñanzas y de las propias instituciones en su conjunto. Dentro de este último grupo, destacan programas como VERIFICA (encaminado a la evaluación de las propuestas de los planes de estudio diseñados por cada centro en consonancia con los objetivos establecidos para la construcción del EEES), AUDIT (dirigido a los centros universitarios para orientarles en el establecimiento de sistemas de garantía interna de calidad), o, para el caso que nos ocupa en este trabajo, DOCENTIA (apoyo a las universidades en el diseño de modelos y procedimientos para valorar la calidad de la actividad docente de sus profesores y favorecer, con ello, la mejor de dicha actividad y de su reconocimiento) ${ }^{2}$.

La evaluación de la actividad docente universitaria, además de estar regulada por ley ${ }^{3}$ en nuestro país desde hace tiempo, alcanza todo su sentido en este nuevo paradigma educativo no sólo como un elemento para la garantía de la calidad de la formación que se imparte en sus estudios sino, igualmente, en otros aspectos de gran importancia en sus políticas internas sobre aspectos tales como la formación, la promoción personal y profesional, la planificación docente o los incentivos económicos de su profesorado (Escudero, Pino y Rodríguez, 2010; Area, Sanabria y González, 2008).

Esta evaluación de la actividad docente universitaria es entendida por el programa DOCENTIA como "la valoración sistemática de la actuación del profesorado considerando su rol profesional y su contribución para conseguir los objetivos de la titulación en la que está implicado, en función del contexto institucional en que se desarrolló”. Además, es igualmente entendida como un proceso de evaluación interna que cada universidad realiza de su profesorado pero en el que pueden participar agentes externos, si así es requerido por alguna instancia externa o si la propia universidad lo considera oportuno. 
Sustenta este proceso la aplicación de un modelo de evaluación ${ }^{4}$ que gira en torno a tres grandes ejes, o "dimensiones", de la actividad docente: la planificación de la docencia, el desarrollo de la enseñanza y los resultados obtenidos. Cada una de estas dimensiones contempla a su vez una serie de subdimensiones, desagregadas éstas en distintos criterios de aplicación como se muestra en la siguiente tabla:

\begin{tabular}{|c|c|c|}
\hline DIMENSIONES & \multicolumn{2}{|l|}{ ELEMENTOS } \\
\hline \multirow{6}{*}{$\begin{array}{l}\text { 1. PLANIFICACIÓN DE } \\
\text { LADOCENCIA }\end{array}$} & \multirow{2}{*}{$\begin{array}{l}\text { 1.1. Organización y coordinación } \\
\text { docentes. }\end{array}$} & $\begin{array}{l}\text { 1.1.1. Modalidades de organización de } \\
\text { la enseñanza. }\end{array}$ \\
\hline & & $\begin{array}{l}\text { 1.1.2. Coordinación con otras actuaciones } \\
\text { docentes. }\end{array}$ \\
\hline & \multirow{4}{*}{$\begin{array}{l}\text { 1.3. Planificación de la enseñanza } \\
\text { y del aprendizaje con relación } \\
\text { a las materias impartidas. }\end{array}$} & $\begin{array}{l}\text { 1.3.1. Resultados de aprendizaje } \\
\text { previstos. }\end{array}$ \\
\hline & & $\begin{array}{l}\text { 1.2.2. Actividades de aprendizaje } \\
\text { previstas. }\end{array}$ \\
\hline & & 1.2.3. Criterios y métodos de evaluación. \\
\hline & & $\begin{array}{l}\text { 1.2.4. Materiales y recursos para la } \\
\text { docencia. }\end{array}$ \\
\hline \multirow{2}{*}{$\begin{array}{l}\text { 2. DESARROLLO DE } \\
\text { LA ENSEÑANZA }\end{array}$} & \multirow{2}{*}{$\begin{array}{l}\text { 2.1. Desarrollo de la enseñanza y } \\
\text { evaluación del aprendizaje. }\end{array}$} & $\begin{array}{l}\text { 2.1.1. Actividades de enseñanza y } \\
\text { aprendizaje realizadas. }\end{array}$ \\
\hline & & $\begin{array}{l}\text { 2.1.2. Procedimientos de evaluación } \\
\text { aplicados. }\end{array}$ \\
\hline \multirow{2}{*}{ 3. RESULTADOS } & \multicolumn{2}{|c|}{ 3.1. Resultados en términos de objetos formativos logrados por los estudiantes. } \\
\hline & \multicolumn{2}{|c|}{ 3.2. Revisión y mejora de la actividad docente: formación e innovación. } \\
\hline
\end{tabular}

Figura 1. Dimensiones de la actividad docente según el modelo DOCENTIA (ANECA)

La aplicación de este modelo que se está haciendo por parte de las distintas comunidades autónomas y sus universidades dependientes, en la mayoría de ellas aún en fase experimental ${ }^{5}$, hace que, en algunos casos, éste sufra adaptaciones y modificaciones, especialmente para darle un mayor peso y protagonismo a la innovación y los programas de mejora de la actividad docente.

Dos de los aspectos más importantes y críticos de este programa están en las fuentes y en los métodos a emplear para la recogida de información pues, si bien en principio se da libertad para que las universidades establezcan lo que les parezca más adecuado y acorde a sus objetivos y procedimientos, se formaliza un modelo 
común, teniendo en cuenta cada una de las tres dimensiones a evaluar y los actores principales intervinientes en este proceso, según ilustra la siguiente tabla:

\begin{tabular}{|l|l|l|l|}
\hline DIMENSIONES & FUENTES Y PROCEDIMIENTOS DE EVALUACIÓN \\
\hline & Profesor & Responsables académicos & Estudiante \\
\hline Planificación & & & \\
\cline { 1 - 1 } Desarrollo & Auto-informe & Informe & Encuesta \\
\cline { 1 - 1 } Resultados & & & \\
\hline
\end{tabular}

Figura 2. Dimensiones, fuentes y procedimientos de evaluación

Sin embargo, el documento base de este programa vuelve a señalar a este respecto que las universidades podrán incorporar aquellas fuentes y procedimientos de recogida de información de elaboración propia (especialmente la que procede de datos producidos por los sistemas informáticos propios de gestión académica). Entre las técnicas adicionales que se señalan a título de ejemplo para la dimensión de "Resultados" se expresa directamente el uso de indicadores de rendimiento.

La Universidad Carlos III de Madrid, en la que se enmarca la presente investigación, como miembro participante en el programa DOCENTIA, y enmarcado dentro de su Sistema de Garantía Interna de Calidad (SGIC) ${ }^{6}$, elaboró su propio modelo de evaluación interna de la docencia (DOCENTIA-UC3M) ${ }^{7}$, siendo presentado en Consejo de Gobierno en octubre de 2007. En él se establecen dos fases de aplicación: una primera con carácter transitorio (prevista, en principio, para un par de cursos), en donde el modelo se adscribe a las tres dimensiones generales y a las fuentes y procedimientos antes señalados; y una segunda fase definitiva, en donde se establece una dimensión final adicional para premiar la colaboración en líneas estratégicas en este campo (cooperación con otras universidades, internacionalización, etc.). Además, en dicha fase final aumentan los baremos y el número de indicadores propuestos, y se reajusta la puntuación asignada a cada una de las subdimensiones y los porcentajes asignados a las distintas dimensiones. $\mathrm{Al}$ respecto del uso de las fuentes y de los procedimientos para la recogida de la información para la elaboración de los informes preceptivos, en el Volumen I del manual que documenta este modelo ${ }^{8}$, se señala textualmente que "los datos cerrados y objetivables (datos de rendimiento en la asignatura, valoración en las encuestas, y cualesquiera otros que se rescaten de procedimientos, sistemas informáticos, etc.) priman frente a opiniones abiertas y difusas". 


\section{ESTUDIO DEL RENDIMIENTO ACADÉMICO EN LA FORMACIÓN UNIVERSITARIA EN LÍNEA}

Si bien para la evaluación de la práctica docente debería ser necesaria la conjugación de una evaluación externa con una autoevaluación institucional (Bolívar, 2008), los modelos evaluativos utilizados en el ámbito de la enseñanza superior suelen poner el acento en el uso de diversos instrumentos para la recogida de la información de interés. Entre éstos destacan, sin duda, los cuestionarios.

En el caso del programa español DOCENTIA, los cuestionarios suelen ir dirigidos, por un lado, a profesores y/o directores de titulación, y vinculados normalmente en los informes que estos actores han de realizar en dicho proceso evaluativo de la planificación de la docencia y el desarrollo de la enseñanza. Por otro lado, éstos están vinculados a la valoración personal que el estudiante realiza de la formación académica recibida, midiendo con ello los resultados alcanzados tanto a nivel particular (una determinada asignatura) como en su conjunto (un cuatrimestre o un curso académico completo). Los datos recogidos a través de estos instrumentos suelen ser procesados informáticamente y sometidos a un tratamiento estadístico.

Los cuestionarios han venido siendo los instrumentos tradicionalmente empleados en las investigaciones educativas de todo tipo (Area, Sanabria y González, 2008; González, 2009). Si bien éstos se enmarcan dentro de las denominadas técnicas de indagación y prospectiva, respetables y perfectamente válidas para la obtención de información cuantificable, no deben ser los únicos instrumentos empleados para la evaluación de los resultados académicos. El carácter subjetivo de las respuestas aportadas en muchas encuestas de opinión dirigidas a los alumnos universitarios, así como la predisposición de éstos hacia ciertos comportamientos o actitudes a la hora de responder, hacen recomendable que los resultados obtenidos deban ser contrastados y complementados con datos objetivos procedentes de otros instrumentos de gestión de la actividad académica (Douglas y Douglas, 2006).

Esta idea parece estar clara en el contexto de la enseñanza on-line mediante el uso intenso de plataformas informáticas para la enseñanza, el aprendizaje constructivista y la evaluación de resultados (Bangert, 2004), al abrirse un inmenso campo de posibilidades para la recogida, tratamiento y análisis de la actividad académica producida, tanto desde el lado del docente como del lado del alumno. En definitiva, hablamos de un creciente interés, tanto a escala nacional como internacional, en el establecimiento de criterios y estándares capaces de medir la calidad de la formación a distancia, muy especialmente en el contexto de la educación superior (Parker, 2004). 
En estos entornos de aprendizaje toman, por tanto, especial relevancia las técnicas y los procedimientos propios de la recolección automática de datos (técnicas de data mining y similares) y el análisis estadístico de los mismos, todo ello adaptado a las particularidades de este nuevo contexto educativo universitario (Romero y Ventura, 2007).

La extracción y análisis de los datos ha cobrado una especial importancia en estos últimos años en múltiples ámbitos de aplicación al poder transformar éstos en información y conocimiento de gran utilidad mediante la aplicación de diversas técnicas. El data mining es un área o campo multidisciplinar en el que confluyen múltiples técnicas y procedimientos tanto de las ciencias de la computación y de las matemáticas como de las ciencias de la información (Klosgen y Zytkow, 2002). Estas técnicas pueden variar dependiendo del campo de aplicación en el que se esté trabajando y de los resultados que se deseen obtener de este análisis. De forma general, se pueden distinguen tres grandes bloques de métodos aplicativos (Ranjan y Malik, 2007):

- Asociaciones: métodos que identifican reglas de afinidad entre las colecciones de datos.

- Clasificación y predicción: estos modelos emplean diversas técnicas de análisis para describir clases de datos y hacer predicciones sobre las mismas.

- Agrupaciones (clustering): técnicas por la que se establecen agrupaciones lógicas de datos según un determinado criterio, creando de este modo segmentos en los mismos con unas determinadas características.

De forma más específica, y siguiendo a Adriaans y Zantinge (2003), entre estas técnicas y herramientas comúnmente utilizadas en la minería de datos podemos encontrar herramientas para la búsqueda y extracción automática de datos, la aplicación de una gran variedad de análisis estadísticos, la visualización y representación gráfica de los datos, las herramientas OALP (Online Analytical Processing), las técnicas de aprendizaje basado en casos, los árboles de decisión, las reglas de asociación, la minería del texto, las redes neuronales o los algoritmos genéticos, entre otras muchas.

En el entorno específico dela enseñanza a distancia mediante el uso de plataformas informáticas, la gran abundancia de datos que son generados de la actividad propia de los alumnos y profesores en sus respectivos cursos resulta una fuente de información de gran riqueza para la toma de decisiones por parte de las autoridades universitarias 
(Hanna 2004; Patkar, 2005; Black, Dawson y Priem, 2008). Su almacenamiento, análisis y tratamiento estadístico resulta, pues, esencial pues con las aplicaciones de todas estas técnicas se pueden llegar a establecer un sinfín de inferencias sobre el comportamiento de un determinado profesor, de un grupo de alumnos, de una determinada asignatura, de un curso completo, de una titulación específica o, por qué no, del comportamiento de toda una institución académica. Y todo ello, revisando los datos que quedan grabados en los ficheros de logs, que registran los accesos a los recursos consultados y a las actividades realizadas en la aplicación informática de la aplicación. Tal es su importancia en la actual que llega a configurarse como una disciplina científica particular bajo la denominación de Educational Data Mining (EDM) (Romero y Ventura, 2010). En este contexto educativo, el proceso del data mining contemplaría las cuatro fases o etapas generales, que según señala Romero son las siguientes (Romero, Ventura y García, 2008):

- Recolección de datos: datos almacenados en las bases de datos del LMS que recogen la actividad de profesores y alumnos en el curso.

- Pre-procesamiento de los datos: los datos son filtrados, eliminando todos aquellos que no serán de utilidad para el estudio emprendido, así como transformados en un formato adecuado para ser tratados.

- Aplicación de los algoritmos: según el estudio emprendido y la información que se desee obtener se aplicarán unos procedimientos y algoritmos u otros, haciendo uso para ello de las herramientas informáticas adecuadas.

- Interpretación, evaluación y obtención de los resultados: mediante la interpretación de los datos obtenidos y la correspondiente información resultante se pueden inferir toda una serie de conclusiones sobre el comportamiento de la muestra analizada de usuarios del sistema.

Si bien es verdad que todas estas técnicas ayudan notablemente a la mejora de los procesos evaluativos de la docencia universitaria, no es menos cierto que confiar únicamente en estas técnicas puede provocar distorsiones en la valoración del comportamiento real de un determinado grupo docente. Son diversos los errores en los que se pueden caer con la aplicación de estas técnicas meramente cuantitativas pero, sin duda, la más grave sea la de "escuchar" únicamente a los datos o, al menos, a los datos obtenidos únicamente de una determinada fuente (Nisbet, Elder y Miner, 2009), como señalábamos al principio de este epígrafe al introducir la técnica del cuestionario. En el caso que nos ocupa, los datos procedentes de los ficheros de actividad de las plataformas LMS pueden provocar 
una visión incompleta de lo que realmente está sucediendo en un determinado curso o titulación si no son contratados con otros datos e informaciones procedentes de otras fuentes de importancia en este tema, tales como las encuestas de evaluación de la docencia tanto de los alumnos como de los profesores (datos concretos y comentarios textuales de los mismos), datos procedentes de los servicios administrativos de gestión académica de la universidad, informes generados en el seno de las comisiones académicas de la titulación, informes de los departamentos y de las facultades, etc.

\section{RECOGIDA Y ANÁLISIS DE LOS DATOS DE ACTIVIDAD GENERADOS POR LA PLATAFORMA MOODLE}

Son muchas las instituciones académicas universitarias de todo el mundo que han venido utilizando la información contenida en los ficheros de actividad de las plataformas de gestión para el aprendizaje a distancia para intentar medir el grado de calidad de las enseñanzas impartidas. En muchos casos, la propia aplicación LMS contempla un módulo estadístico capaz de presentar y representar gráficamente determinados datos de la actividad del curso, tanto de carácter general como de aspectos concretos del mismo. En otros casos y para otros propósitos de mayor calado, en los que el acceso a la totalidad de los datos registrados en el sistema es requerido, se hace necesario acceder directamente a los ficheros log del sistema, bien de los propios del servidor, del cliente y/o del proxy (Gu et al., 2008). A partir de los datos registrados en estos ficheros, y con la ayuda de programas informáticos de análisis y tratamiento de datos (principalmente de carácter estadístico) se obtienen las inferencias deseadas.

Es el caso específico de la plataforma Moodle [http://www.moodle.org/], ampliamente utilizada en todo el mundo como herramienta base para la docencia universitaria, tanto presencial, a distancia o, como es el caso que nos ocupa, en modelos de estudios semipresenciales. Esta plataforma no contempla un módulo estadístico como tal donde el profesor, el responsable académico o la autoridad competente en la materia pueda obtener de forma directa información estadística ya elaborada. Por este motivo, será necesario acudir a los ficheros de actividad del propio sistema. A diferencia de lo que ocurre con otras aplicaciones similares, Moodle no almacena estos logs en ficheros de texto sino que lo hace directamente en las bases de datos propias del sistema (Rice, 2006). Ello significa que cada acción que el usuario realiza queda representada en la tabla de la base de datos en cuestión como una fila o registro de la misma. Cada campo de dicho registro identifica la información que queda almacenada (identificador, fecha y hora, usuario, dirección IP desde la que 
accede, curso, módulo activado, la acción realizada e información adicional relativa al recurso o actividad accedido por el usuario). Así, y dependiendo del espacio de la aplicación en el que nos encontremos, Moodle nos ofrecerá abundante información de carácter estadístico de un tipo u otro.

De forma sectorial, esta aplicación ofrece información de carácter estadístico de interés para la evaluación del curso en muchas de sus áreas y pantallas, desde la simple vista del listado de alumnos matriculados en el curso (donde ya se está informando del tiempo que hace desde que cada usuario accedió por última vez) hasta información más compleja y detallada en todas las tareas y actividades programadas, bien de forma puntual en cada tarea, bien de forma general por la toda la actividad realizada en el curso por un determinado alumno (diagrama de informe, informe completo, registros de hoy, todas las entradas, estadísticas y calificación), como se puede apreciar en la siguiente figura.

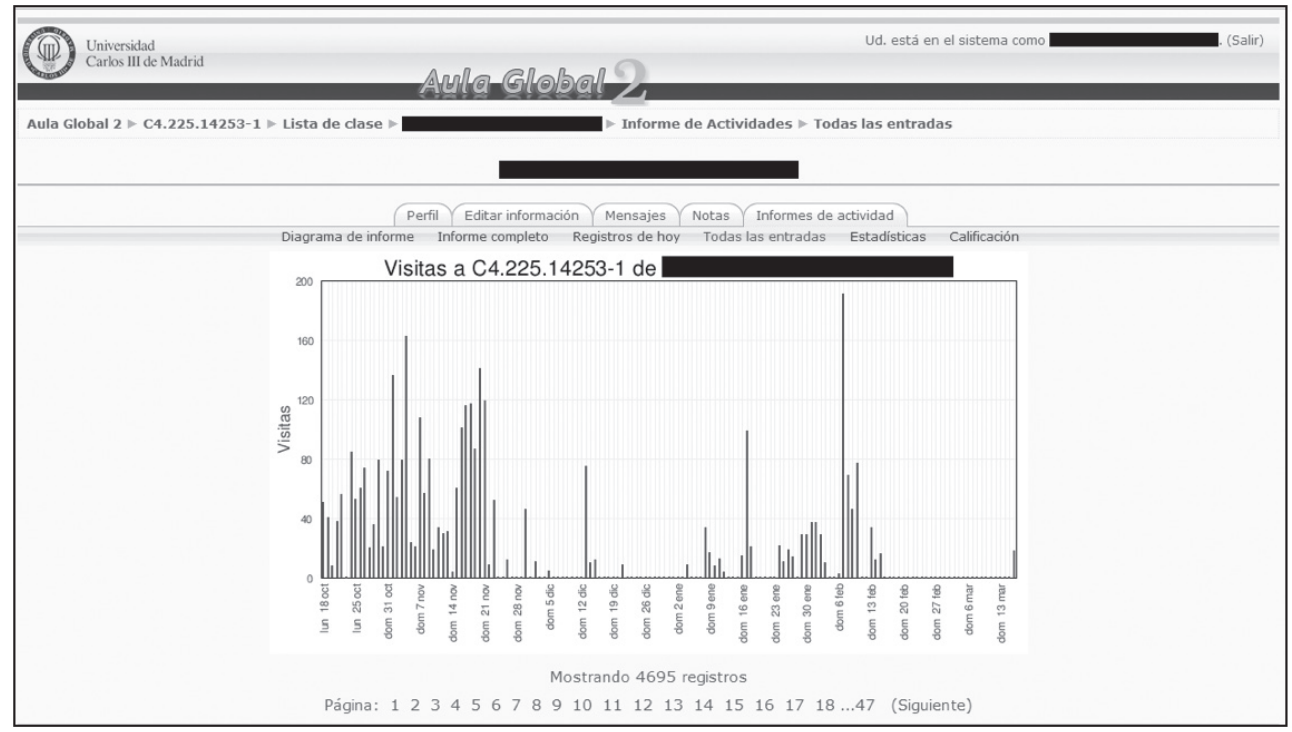

Figura 3. Acceso al informe de actividad de un alumno del curso en particular

Sin embargo, Moodle permite centralizar el acceso a todos los datos de carácter estadístico desde un único sitio: los Informes. Se trata de una opción del bloque de Administración de acceso exclusivo del docente del curso en el que puede establecer la combinación de una serie parámetros (todos los participantes o uno en particular, una fecha en concreto o para el total del tiempo de duración del curso, todas las actividades o una en particular, y todas las acciones o una en particular) para obtener 
la información cuantitativa de carácter global o sectorial que se desea. En cualquier caso, y sea la combinación que sea la establecida, el sistema ofrece la posibilidad de o bien visualizar los datos obtenidos en la página de la propia aplicación o descargarlos en un archivo en un determinado formato (texto, ODS o .xls), como se aprecia en la siguiente figura:

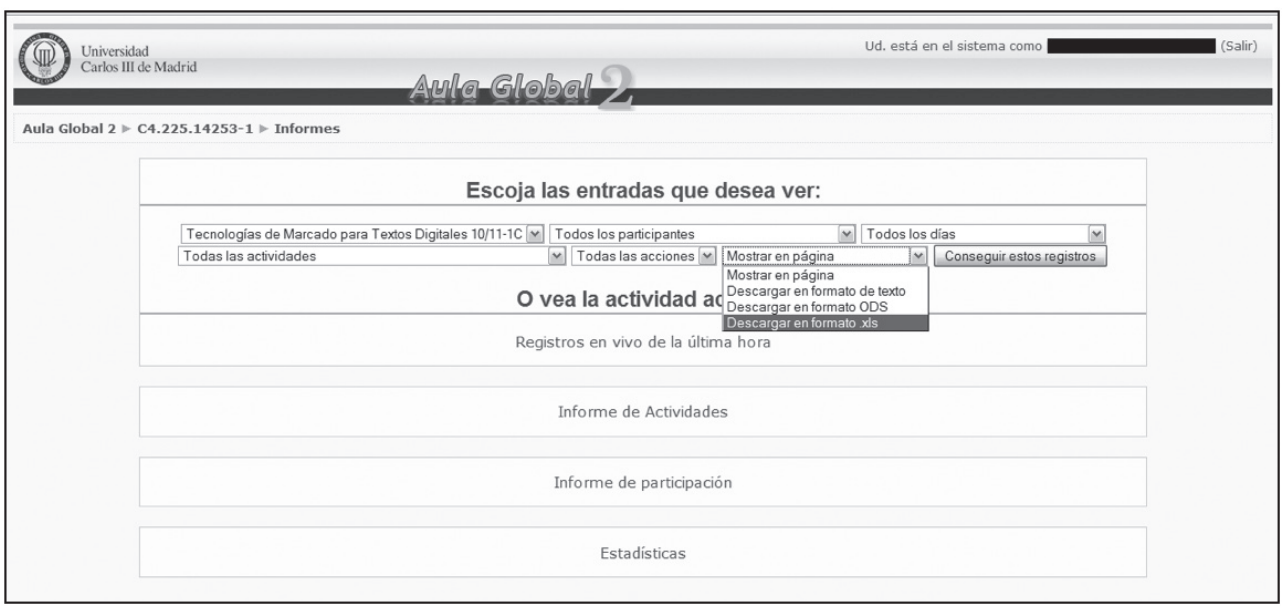

Figura 4. Página de acceso a los informes de actividad del curso

Es, sin duda, esta posibilidad de extracción y descarga de los datos de actividad del curso de Moodle la función de mayor interés para las investigaciones que sobre su uso se están realizando en la actualidad en el seno de nuestro departamento universitario. Con ello, es posible obtener todo el material en bruto para su posterior procesamiento y tratamiento mediante el uso de herramientas informáticas de carácter estadístico.

La combinación de los posibles valores que puede tomar el campo de "acciones" llevadas a cabo por el alumno o profesor en el curso, que, de forma general, ofrece las opciones de "todas las acciones", "vista", "añadir", "actualizar", "borrar" y "todos los cambios" pero en el que se pueden descubrir muchas más acciones derivadas de estos valores en el propio fichero log (más de medio centenar de acciones específicas), junto con el resto de campos (especialmente con el relativo a "Todas las acciones" o recursos existentes en el curso), producirá una salida de datos tan genérica o específica como deseemos. Así, por ejemplo, optando por dejar todos los campos existentes en la página de la generación de informes en su valor más global, se obtendría la visión más completa de la actividad del conjunto de profesores y alumnos del curso en cuestión, desde el primer día de apertura del mismo hasta el momento de generación del 
informe (normalmente a la finalización del curso). La manipulación e interpretación de los datos obtenidos en dicho informe, como señalábamos anteriormente, mediante las herramientas informáticas de carácter estadístico empleadas pueden ayudar en la búsqueda de respuestas sobre las dinámicas y comportamientos, tanto grupales como individuales, de la actividad académica de los estudiantes en este entorno informatizado y a distancia de formación universitaria.

Si bien son innumerables las experiencias sobre el uso de la plataforma Moodle en el ámbito de la educación universitaria en España que podemos encontrar en la literatura científica (González, 2006; Ruiz y Romero, 2008; Area, Sanabria y González, 2008; Herradón et al, 2009), no lo es tanto, ni mucho menos, al respecto del tema que nos ocupa, encontrando la mayoría de las experiencias fuera de nuestro país. Sin embargo, son destacables las investigaciones sobre el tema llevadas a cabo en estos últimos años en el Departamento de Informática de la Universidad de Córdoba (España) a través del profesor C. Romero y su equipo de investigación (Romero y Ventura, 2007; Romero, Ventura y García, 2008; Romero et al., 2009).

En estos estudios, de carácter netamente empíricos, se exponen las investigaciones que desde este departamento universitario se han venido realizando con los datos procedentes de los logs de la plataforma Moodle, agrupando las aplicaciones que se derivan de las técnicas de la minería de datos en diferentes categorías: estadísticas (como elemento de inicio de la evaluación), visualización (representación gráfica de los datos obtenidos), agrupación (mediante técnicas de clustering se representan las agrupaciones de los objetos tratados), clasificación (sistemas de análisis de clasificación o discriminación que permiten establecer predicciones sobre posibles comportamientos de los estudiantes o grupos de éstos), reglas de asociación (que expresan las correlaciones existentes entre los valores de los registros de la base de datos con las condiciones y su frecuencia de ciertas actividades del curso) y otras tantas técnicas ya señaladas con anterioridad. Para realizar todas estas operaciones, han venido utilizando programas informáticas específicos, tanto comerciales como de software libre, destacando especialmente en este último grupo los sistemas Weka [http://www.cs.waikato.ac.nz/ml/weka/], desarrollado por la Universidad de Waikato (Nueva Zelanda) y Keel [http://www.keel.es/], desarrollado por un grupo de universidades españolas (Granada, Jaén, Córdoba, Oviedo, Ramón Llull y Huelva). En ambos casos estos dos productos han sido implementados en lenguaje Java y emplean el mismo formato de representación externa del conjunto de datos (ficheros ARFF). 
En todos estos trabajos se concluye resaltando la utilidad de estas técnicas al tratamiento y análisis de los datos procedentes de los ficheros de actividad de estas plataformas de aprendizaje a distancia pero señalando, en el caso de la plataforma Moodle, la necesidad de construir herramientas informáticas específicas (o módulos que se acoplen al núcleo central de este LMS), con interfaces sencillas e intuitivas capaces de ser manejas por los profesores o gestores de cursos.

\section{CONCLUSIONES}

La necesidad de diversidad y de flexibilidad formativa que requiere el nuevo contexto europeo en la ordenación de las enseñanzas universitarias obliga a la autoridades académicas nacionales, así como a las propias universidades del país, a adaptar o ampliar, según los casos, la oferta de titulaciones a nuevas modalidades o tipos de enseñanza que vayan más allá de la tradicional clase magistral de carácter presencial. Este requerimiento se hace aún más evidente en el actual contexto tecnológico en el que nos encontramos, donde las tecnologías propias de la Internet y las derivadas de la telefonía móvil posibilitan crear nuevos contextos de enseñanza y aprendizaje en los que la comunicación e interactuación entre profesores y alumnos se realiza en un entorno electrónico virtual. Surgen con ello nuevos términos y modalidades formativas en este contexto (b-learning, e-learning, mobile learning, etc.) al que los docentes debemos adaptarnos pero que, de igual modo, las autoridades académicas han de dar respuestas a nuevos interrogantes que van surgiendo.

Uno de ellos es, sin duda, la adaptación de los actuales sistemas de evaluación del profesorado y de la calidad de la docencia en el marco de los denominados Sistemas de Garantía de la Calidad puestos en marcha por las distintas universidades del país y bajo la supervisión de la ANECA. Así, programas nacionales como VERIFICA, AUDIT o, en el caso que nos ocupa, DOCENTIA deben de ir adaptándose a estos nuevos tiempos, requerimientos y modalidades formativas en los que el uso de las denominadas plataformas educativas (LMS) toma un papel central como espacio virtual de encuentro y desarrollo de la docencia.

Sin embargo, y a pesar de ese uso cada vez más intenso de estas plataformas por parte de la comunidad universitaria, los modelos de evaluación de la actividad docente se siguen basando en información recogida de carácter puramente administrativa (altas y bajas de alumnos en un curso, número de alumnos aprobados, que abandonan el curso, etc.) o bien con una alta subjetividad (encuestas a alumnos y autoinformes del profesor y del responsable académico). Se hace, por tanto, necesario incorporar al estudio, análisis y evaluación de la formación universitaria actual todos los datos 
que estos sistemas van generando dentro de cada asignatura, curso y titulación, de tal modo que puedan mostrar de forma más objetiva y completa las dinámicas y los comportamientos producidos por los actores intervinientes en estos procesos educativos. Con ello, es posible intuir de forma más precisa cuestiones relativas al funcionamiento tanto general como particular de un curso (su grado de actividad y seguimiento, su diversidad curricular, el nivel de comunicación entre alumnos y profesor, tasa progresiva de abandono, etc.).

Dada la gran cantidad de datos de todo tipo que estos sistemas producen a través de sus registros de actividad (ficheros log), se hace necesario el uso de técnicas y procedimientos especiales procedentes de diversas áreas del conocimiento, especialmente de la informática y de la estadística. La minería de datos (data mining) aplicada a este contexto educativo, el denominado Educational Data Mining (EDM), está alcanzando en estos años un gran interés pues las inferencias que se pueden llegar a alcanzar pueden ayudar notablemente a encontrar indicadores más adecuados y contextualizados en estos entornos formativos tan informatizados.

En el caso específico de la plataforma Moodle, de gran implantación en universidades de todo el mundo, al ser un desarrollo bajo licencia de software abierto, parece claro que el uso y el tratamiento estadístico de los datos generados a través de sus ficheros log debe conducir al establecimiento de ciertos indicadores que permitan complementar a los que actualmente se emplean en los sistemas de evaluación de la actividad docente, al ser éstos el mejor registro de lo que sucede día a día en el seno de esas aulas virtuales.

\section{NOTAS}

$1 \quad$ Véanse la Ley Orgánica 4/2007, de 12 de abril, por la que se modifica la Ley Orgánica 6/2001, de 21 de diciembre, de Universidades, disponible en http://www.boe.es/boe/ dias/2007/04/13/pdfs/A16241-16260.pdf, y el Real Decreto 1393/2007, de 29 de octubre, por el que se establece la ordenación de las enseñanza universitarias oficiales, disponible en http://www.boe.es/boe/dias/2007/10/30/pdfs/A44037-44048.pdf

2 El programa DOCENTIA toma como referencia los criterios y las directrices definidas, principalmente, en el espacio europeo de educación superior, a través del trabajo de la European Association for Quality Assurance in Higher Education (ENQA) [ http://www.enqa.eu/ ], así como, en el ámbito de aplicación norteamericano, a través de los estándares establecidos por el Joint Committee on Standards for Educational Evaluation [http://www.jcsee.org/].

3 Establecida en el artículo 43.3 de la Ley Orgánica 6/2001 de 21 de diciembre, de Universidades (LOU), al señalar que las universidades dispondrán de procedimientos para la evaluación periódica del rendimiento docente y científico del profesorado. 
Texto electrónico disponible en http://www.boe.es/boe/dias/2001/12/24/pdfs/ A49400-49425.pdf

4 Disponible en http://www.aneca.es/media/215769/docentia modelo 070302.pdf

5 Según datos aportados por Isabel Belmonte en su presentación "El modelo DOCENTIA y su fase de implantación" en el marco de la Jornada dirigida a las Comisiones de Evaluación de la Universidad de Vigo (14/01/2010) el 86\% de las universidades existentes en el Sistema Universitario Español participan en el programa DOCENTIA pero sólo un 46\% de ellas ha comenzado en el curso 2008/2009 su fase de implantación. Ver en http://www.acsug.es/webs/ficheros/ibo.pdf

6 Los primeros resultados obtenidos de este sistema tras la implantación de los nuevos estudios de Grado fueron presentados por la Vicerrectora de Postgrado y Calidad en julio de 2009 en la Universidad de Burgos en el marco de los "Encuentros sobre Calidad en la Educación Superior". Véase en http://www.kongresuak.ehu.es/p275content/eu/contenidos/noticia/ubu calidad sistemas inform/eu encuentr/ adjuntos/Burgos UC3M Carmen 5.pdf

7 Véase en http://www.uc3m.es/portal/page/portal/organizacion/secret general/ normativa/pdi/meritos doc invest/Manual\%20Docentia.Anexo\%20resumen\%20e jecutivo.pdf

8 Véase en http://www.uc3m.es/portal/page/portal/organizacion/secret general/ normativa/pdi/meritos doc invest/Manual Docentia Volumen I.pdf

\section{REFERENCIAS BIBLIOGRÁFICAS}

Ahn, J. Y.; Han, K. S. (2005). Web-based education: characteristics, problems, and some solutions. International Journal of Innovation and Learning, vol. 2, $\mathrm{n}^{\mathrm{O}} 3$, (274-282).

Area Moreira, M. (2006). Los ECTS y el aula virtual Moodle: Análisis de una experiencia docente universitaria semipresencial. (79-92). En: Castro Sánchez, J. J. (Coord.). Docencia universitaria a través de entornos virtuales de enseñanza-aprendizaje. Las Palmas de Gran Canaria: Universidad de Las Palmas de Gran Canaria, Servicio de Publicaciones.

Area Moreira, M.; Sanabria Mesa, A. L.; González Afonso, M. (2008). Análisis de una experiencia de docencia universitaria semipresencial desde la perspectiva del alumnado. RIED. Revista Iberoamericana de Educación a Distancia, vol. 11, $\mathrm{n}^{\mathrm{O}} .1$, (231-254). [en línea] Disponible en: http://www.utpl. edu.ec/ried/images/pdfs/volumen11/ manuel-area.pdf (consulta 2011, 24 de junio).

Bangert, A. W. (2004). The seven principles of good practice: A framework for evaluating on-line teaching. Internet and Higher Education, $\mathrm{n}^{0}$ 7, (217-232).

Black, E. W.; Dawson, K.; Priem. J. (2008). Data for free: Using LMS activity logs to measure community in online courses. Internet and Higher Education, $\mathrm{n}^{\mathrm{O}}$ 11, (65-70).

Bolívar, A. (2008). Evaluación de la práctica docente. Una revisión desde España. Revista Iberoamericana de Evaluación Educativa, vol. 1, no. 2, (56-74). [en línea] Disponible en: http://rinace. net/riee/numeros/vol1-num2/art4.pdf (consulta 2011, 24 de junio).

Chen, C. M.; Chen, M. C. (2009). Mobile formative assessment tool based on data 
mining techniques for supporting webbased learning. Computers \& Education, $\mathrm{n}^{0} 52,(256-273)$.

Díaz-Antón, G.; Pérez, M. (2005). Hacia una ontología sobre LMS. Proceeding VII Jornadas Internacionales de las Ciencias Computacionales. Universidad de Colima, Colima, México. [en línea] Disponible en: http://www.lisi.usb.ve/publicaciones/ 02\%20calidad\%20sistemica/calidad 59.pdf (consulta 2011, 24 de junio).

Douglas, J.; Douglas, A. (2006). Evaluating Teaching Quality. Quality in Higher Education, vol. 12, $\mathrm{n}^{0}$ 1, (3-13). [en línea] Disponible en: http://www.ljmu. ac.uk/BLW/BLW Facultytopleveldocs/ Evaluating Teaching Quality.pdf (consulta 2011, 24 de junio).

Escudero Escorza, T.; Pino Mejías, J. L.; Rodríguez Fernández, C. (2010). Evaluación del profesorado universitario para incentivos individuales: revisión metaevaluativa. Revista de Educación, $\mathrm{n}^{\mathrm{o}}$. 351, (513-537). [en línea] Disponible en: http://www.revistaeducacion.mec. es/re351/re351 21.pdf (consulta 2011, 24 de junio).

Gabroveanu, M.; Ion-Mircea, D. (2008). Extracting Semantic Annotations from Moodle Data. CEUR Workshop Proceedings, $\mathrm{n}^{\mathrm{o}}$ 418. [en línea] Disponible en: http://sunsite.informatik. rwth-aachen.de/Publications/CEURWS/Vol-428/paper1.pdf (consulta 2011, 24 de junio).

González, I. (2009). La autopercepción de la formación universitaria: evaluación y calidad. Revista Iberoamericana de Evaluación Educativa, vol. 2, nº . 2, (157170).

González Mariño, J. C. (2006). B-Learning utilizando software libre, una alternativa viable en Educación Superior. Revista Complutense de Educación, vol. 17, $\mathrm{n}^{\mathrm{0}}$ 1, (121-133).
Gu, R.; Zhu, M.; Zhao, L.; Zhang, N. (2008). Interest mining in virtual learning environments. Online Information Review, vol. 32, $\mathrm{n}^{\circ}$ 2, (133-146).

Hanna, M. (2004). Data mining in the elearning domain. Campus - Wide Information Systems, vol. 21, $\mathrm{n}^{0}$ 1, (2934).

Herradón Díez, R.; Blanco Cotano, J.; Pérez Yuste, A.; Sánchez Fernández, J. A. (2009). Experiencias y metodologías "blearning" para la formación y evaluación en competencias genéricas en Ingeniería. La Cuestión Universitaria, $\mathrm{n}^{\mathrm{O}} 5$, (3345).

Iribarren Maestro, I.; Rodríguez Mateos, D.; Bueno de la Fuente, G.; Martín Galán, B.; Azcárate Aguilar-Amat, P.; García Zorita, C. (2008). A Blended Learning Model for Two Library and Information Science Degrees at Carlos III University of Madrid: A Case Study. International Conference of Education, Research and Innovation. Valencia: IATED.

Klosgen, W.; Zytkow, J. (2002). Handbook of data mining and knowledge discovery. New York: Oxford University Press.

Nisbet, R.; Elder, J.; Miner, G. (2009). Handbook of statistical analysis and data mining applications. Oxford: Academic Press/Elsevier.

Oliveros Martín-Vares, L. (2006). Identificación de competencias: una estrategia para la formación en el Espacio Europeo de Educación Superior. Revista Complutense de Educación, vol. 17, $\mathrm{n}^{\circ}$ 1, (101-118). [en línea] Disponible en: http://revistas.ucm.es/edu/11302496/ articulos/RCEDo606120101A.PDF (consulta 2011, 24 de junio).

Parker, N. K. (2004). The quality dilema in online education. En: Anderson, T.; Elloumi, F.(Eds.). Theory and Practice of Online Learning. Athabasca: Athabasca University. [en línea] Disponible en: http://www.aupress.ca/books/120146/ ebook/13 Anderson 2008 Parker- 
Online Courses.pdf (consulta 2011, 24 de junio).

Patkar, V. N. (2005). Data mining applications in library and academic institutions. Informations Studies, vol. $11, \mathrm{n}^{0} 3,(145-156)$.

Ranjan, J.; Malik, K. (2007). Effective educational process: a data-mining approach. VINE: The Journal of Information and Knowledge Management Systems, vol. 37, $\mathrm{n}^{\mathrm{O}} 4$, (502-515).

Rice, W. H. (2006). Moodle. E-Learning Course Development. Birmingham: Packt Publishing.

Romero, C.; Ventura, S. (2010). Educational Data Mining: A Review of the State of the Art.IEEETransactions on Systems, Man, and Cybernetics - Part C: Applications and Reviews, vol. 40, $\mathrm{n}^{\circ}$ 6, (601-618).

Romero, C.; González, P.; Ventura, S.; del Jesús, M. J.; Herrera, F. (2009). Evolutionary algorithms subgroup discovery in e-learning: A practical application using Moodle data. Expert Systems with Applications, $\mathrm{n}^{\circ} 36,(1632-$ 1644).
Romero, C.; Ventura, S.; García, E. (2008). Data mining in course management systems: Moodle case study and tutorial. Computers \& Education, $\mathrm{n}^{0}$ 51, (368384).

Romero, C.; Ventura, S. (2007). Educational data mining: A survey from 1995 to 2005. Expert Systems with Applications, $\mathrm{n}^{\mathrm{o}}$ $33,(135-146)$.

Ruiz, I.; Romero, S. (2008). Moodle: una herramienta eficazaplicadaala enseñanza de las prácticas, en el área de electrónica y arquitectura de los computadores. VIII Congreso de Tecnologías Aplicadas a la Enseñanza de la Electrónica. Zaragoza. [en línea] Disponible en: http://taee. euitt.upm.es/Congresosv2/2008/ papers/2008S3C05.pdf (consulta 2011, 24 de junio).

Torrecilla Murillo, F. J. (2008). La Evaluación del profesorado universitario en España. Revista Iberoamericana de Evaluación Educativa, vol. 1, $\mathrm{n}^{\mathrm{o}}$. 3, (29-45). [en línea] Disponible en: http://rinace.net/ riee/numeros/vol1-num3 e/art3.pdf (consulta 2011, 24 de junio).

\section{PERFIL ACADÉMICO Y PROFESIONAL DE LOS AUTORES}

Bonifacio Martín Galán. Profesor del Departamento de Biblioteconomía y Documentación de la Universidad Carlos III de Madrid. Miembro del grupo de investigación TECNODOC para la aplicación de las tecnologías de la información y las comunicaciones en bibliotecas, archivos y centros de documentación. Hasta fechas recientes ha ostentado el cargo académico de Vicedecano de la Facultad de Humanidades, Comunicación y Documentación en el campus de Colmenarejo, impulsando desde ahí los estudios académicos de Biblioteconomía y Documentación en modalidad semipresencial.

E-mail: bmartin@bib.uc3m.es 


\section{DIRECCIÓN DEL AUTOR:}

Universidad Carlos III de Madrid

Campus de Colmenarejo

Avda. de la Universidad Carlos III, $\mathrm{n}^{0} 22$

28270 Colmenarejo, Madrid - España

David Rodríguez Mateos. Profesor del Departamento de Biblioteconomía y Documentación de la Universidad Carlos III de Madrid. Miembro del grupo de investigación TECNODOC para la aplicación de las tecnologías de la información y las comunicaciones en bibliotecas, archivos y centros de documentación. Hasta fechas recientes ha formado parte del grupo de expertos de la $\mathrm{UC}_{3} \mathrm{M}$ en el uso de la plataforma Moodle para su aplicación en la docencia universitaria, así como del Equipo de Coordinación Pedagógica del Departamento.

E-mail: david.rodriguez@uc3m.es

DIRECCIÓN DEL AUTOR:

Universidad Carlos III de Madrid

Campus de Getafe

C/ Madrid, $\mathrm{n}^{\mathrm{0}} 126$

28903 Getafe, Madrid, España

Fecha de recepción del artículo: 07/09/11

Fecha de aceptación del artículo: 15/10/11

Como citar este artículo:

Martín Galán, B.; Rodríguez Mateos, D. (2012). La evaluación de la formación universitaria semipresencial y en línea en el contexto del EEES mediante el uso de los informes de actividad de la plataforma Moodle. RIED. Revista Iberoamericana de Educación a Distancia, volumen 15, nº 1, pp. 159-178. 